\title{
GMR
}

\section{Screening of genes related to ovarian development in the swimming crab, Portunus trituberculatus, by suppression subtractive hybridization}

\author{
Z.B. $\mathrm{Yu}^{1,2}$, C.K. Mu${ }^{1,2}$, W.W. Song ${ }^{1,2}$, R.H. Li ${ }^{1,2}$, Y.E. Chen ${ }^{1,2}$ and C.L. Wang ${ }^{1,2}$ \\ ${ }^{1}$ School of Marine Science of Ningbo University, Zhejiang, Ningbo, China \\ ${ }^{2}$ Collaborative Innovation Center for Zhejiang Marine High-efficiency and Healthy \\ Aquaculture, Zhejiang, Ningbo, China \\ Corresponding author: C.L. Wang \\ E-mail: wangchunlin@nbu.edu.cn
}

Genet. Mol. Res. 14 (4): 18675-18686 (2015)

Received August 30, 2015

Accepted October 6, 2015

Published December 28, 2015

DOI http://dx.doi.org/10.4238/2015.December.28.16

ABSTRACT. The swimming crab, Portunus trituberculatus, is an important marine animal and is widely cultured in China. In the present study, suppression subtractive hybridization was applied to identify the differentially expressed genes in the ovaries of mature and immature $P$. trituberculatus. One hundred and seventy six expressed sequence tag (ESTs) were identified, of which 100 were down-regulated, and 76 up-regulated. BLAST analysis identified 51 unigenes, of which 27 were down-regulated, and 24 up-regulated. Quantitative real-time reverse transcriptase polymerase chain reaction results indicated that the $\mathrm{SSH}$ technique is valuable in screening genes related to ovarian development. Genes identified in this study encoded proteins corresponding to a wide range of functions and included immune response protein, transcription initiation factor, metabolic proteins, chromosome, histone h3, ovarian development-related protein, and vitellogenin. In addition, 64 metabolic pathways were annotated in differentially expressed ESTs by using the 
Kyoto Encyclopedia of Genes and Genomes pathway. Four annotated pathways (oxidative phosphorylation, carbon metabolism, fatty acid degradation, and protein digestion and absorption) appeared to be involved in ovarian development. In ontology analysis, $5.83 \%$ of the cellular process genes in reverse subtraction cDNA library are involved in reproduction, and $5.88 \%$ involved in developmental process. In up-regulated genes, myosin II-expressed polehole-like protein; histone h3; ovigerous-hair stripping substance; peritrophin 48; and ovarian development-related protein appeared to be involved in ovarian development. Identification of differentially expressed genes in the mature and immature ovary of the swimming crab provides new insights for further studies on the mechanism underlying ovarian development in this species.

Key words: Portunus trituberculatus; Ovarian development; Suppression subtractive hybridization

\section{INTRODUCTION}

Portunus trituberculatus is a commercially significant crab species and is widespread throughout Korea, Japan, China, and Southeast Asia. In China, P. trituberculatus is distributed throughout the marginal seas of China, including the Bohai, Huanghai, East China, and South China seas. Due to its high nutritional value, $P$. trituberculatus is a species that is intensively explored commercially in China. With the expanding scale of $P$. trituberculatus culture, more and more mature female crabs are cultured to meet the requirement for larval crabs. Ovarian maturation is therefore a critical step in reproduction of the species within culture systems. Programs of differential gene expression mediate eukaryotic biological processes, such as cellular growth and organogenesis. Molecular mechanisms of $P$. trituberculatus reproduction remain largely unknown, and mechanisms of ovarian development are worthy of being explored.

Genes related to ovarian development in the Chinese shrimp, Fenneropenaeus chinensis, have been identified by suppression subtractive hybridization (SSH) (Xie et al., 2010). Several genes have been characterized in $P$. trituberculatus, but most of these are immune-related, and few are associated with sex or gonad development (Liu et al., 2011). From a physiological and behavioral standpoint, reproduction in crustaceans is a complex process (Elner and Beninger, 1992). In the swimming crab, it had been observed that ovarian development is dependent on mating (Wu et al., 2007).

Identification of genes that are differentially expressed in the ovary, at the level of transcription, may help to elucidate mechanisms of ovarian maturation, and facilitate the development of novel methods for reproduction control in $P$. trituberculatus. Subtractive cDNA hybridization has been shown to be a powerful technique for the identification and isolation of cDNA of differentially expressed genes, and several forms of subtractive hybridization have been reported (Duguid and Dinauer, 1990). The polymerase chain reaction (PCR)-based SSH technique was developed for rapid and sensitive comparisons of mRNA expression patterns between 'tester' and 'driver' populations, and has been applied to many molecular genetic and positional cloning studies for the identification of disease, developmental, tissue-specific, or other differentially expressed genes (Diatchenko et al., 1996). In the present study, PCR-based SSH was applied to 
screen genes associated with ovarian maturity in the swimming crab, providing a useful molecular resource for further investigation of mechanisms of ovarian development in this species.

\section{MATERIAL AND METHODS}

\section{Animals, culturing, and treatment}

Two hundred $P$. trituberculatus, with a sex ratio of $1: 1$, were cultured at Xinyi Corporation of Ningbo, China. Each crab was cultured in a separate plastic basket, except for mating. To control ovarian maturation, one group, containing 50 female crabs, were allowed to mate (the tester group); a second group was not mated (the driver group). Crabs in these two groups were cultured for three months under the same conditions, before the tester group was mated. Ovarian maturity was then assessed by collection of ovarian tissues for RNA isolation and construction of differential expression cDNA libraries between the tester and driver groups.

\section{Total RNA and mRNA isolation}

Crabs were anesthetized by chilling, then euthanized and ovarian tissues were harvested from females for total RNA extraction, which was performed immediately using Trizol reagent, according to the manufacture protocol (Invitrogen, USA). Integrity of the extracted RNA was examined by electrophoresis on denaturing, formaldehyde $1 \%$ agarose/EtBr $(\mathrm{w} / \mathrm{v}) \mathrm{gel}$.

\section{Construction of SSH libraries and dot blotting}

A differential expression cDNA library was constructed with SSH using a PCR-select ${ }^{\mathrm{TM}}$ cDNA subtraction kit, according to the manufacturer protocol (Clontech, Tokyo, Japan). For forward subtraction, cDNA from mature ovary was used as the tester, and cDNA of immature ovary as the driver. Conversely, cDNA from immature ovary was used as the tester, and cDNA from mature ovary as the driver for reverse subtraction, in order to maximize the sensitivity of the screening kit. Double stranded tester and driver cDNA were digested separately in $R$ sal at $37^{\circ} \mathrm{C}$ for $1.5 \mathrm{~h}$, and then purified prior to tester ligation to adaptors 1 and $2 \mathrm{R}$ (provided in the kit). Each sample was incubated at $98^{\circ} \mathrm{C}$ for $1.5 \mathrm{~min}$ before hybridization at $68^{\circ} \mathrm{C}$ for $8 \mathrm{~h}$. Then, the two samples from the first hybridization were combined, and fresh denatured driver DNA added to further enrich for differentially expressed sequences. Then, cDNAs were directly inserted into the pMD18-T cloning vector (TaKaRa, Japan), transformed into E. coli DH5 $\alpha$-competent cells, and cultured in LB medium (containing $50 \mu \mathrm{g} / \mathrm{mL}$ ampicillin). Dot blotting programs were conducted according to the manufacturer instructions for the PCR-select cDNA subtraction kit.

\section{Sequencing and analysis of expressed sequence tags (ESTs)}

Clones were sequenced on an ABI3730XL automated sequencer (Applied Biosystems, Norwalk, USA). DNA from E. coli, vector, and adapter sequences were removed. Then, sample sequences were screened against the NCBI database (http://www.ncbi.nlm.nih.gov/tools/vecscreen/ univec/). Sequence similarities were searched by local BLASTx and BLASTn analyses against the non-redundant (nr) protein. Gene classification and function were determined by gene ontology (GO) 
mapping, provided by the GO website (http://geneontology.org/). Metabolic pathways were annotated using KEGG Automatic Annotation Server (KAAS) (http://www.genome.jp/tools/kaas/).

\section{Real-time RT-PCR analysis}

Ovarian tissue from crabs in mature and immature groups (both $N=10$ ) were collected and preserved in liquid nitrogen before RNA extraction. Total RNA was extracted as outlined above. Primers for quantitative real-time RT-PCR were designed on the basis of unique gene sequence (Table 1). Transcription levels of eight unique genes were analyzed, using $\beta$-actin as an internal gene control.

Table 1. Primers for determining transcription levels of selected genes.

\begin{tabular}{lllc}
\hline Primer & Forward (5'-3') & Reverse (5'-3') & Length of product (bp) \\
\hline $\begin{array}{l}\text { B-actin } \\
\text { Up-regulated genes }\end{array}$ & TCACACACTGTCCCCATCTACG & ACCACGCTCGGTCAGGATTTTC & 114 \\
$\quad$ Low-Density Lipoprotein Receptor (LDLR) & CCACCATAACACCGCTCACCA & TCTGGCTGGCAGCTCAGGAAT & 115 \\
$\quad$ Ovary development-related protein (ODR) & GCTGTTGTTTGGGCTTTGGTA & GTGGTAGTTAGTGGTGGCGCA & 296 \\
$\begin{array}{l}\text { Polehole-like protein (PLP) } \\
\quad \text { Vitellogenin (VT) }\end{array}$ & TGCTGCCAAACTGTCCTTCAT & TTAATTGCCCAGGTATCGTCAT & 240 \\
Down-regulated genes & & & 300 \\
$\quad$ Comm domain-containing protein 2 (CDC) & CAGTGAGGTGGAGGTCAAGGA & TCTCGGAACCAGCATCTAGGGA & 164 \\
$\begin{array}{l}\text { Hemocyanin subunit 6(HC) } \\
\text { Liver fatty acid binding protein (LFAB) }\end{array}$ & CTGGGTAAACTGTCTCCTGGA & AAGCTGCTGTGGTACTCATCGA & 162 \\
Peroxiredoxin 6 (PRX6) & ACAAATGGGGCATCATCTTCT & TGGTAAAGGTGCCAGTCAAGGT & 124 \\
\hline
\end{tabular}

\section{RESULTS}

\section{SSH library construction and overall features of differentially expressed clones}

Clones $(N=200)$ from the SSH library, constructed from ovary from mature and immature female crabs, were randomly picked and sequenced. In total, 176 differentially expressed cDNA clones were identified. Further analysis revealed that in mature ovary, 57.95\% of SSH clones were down-regulated, and $42.05 \%$ were up-regulated.

\section{Sequencing and analysis of ESTs}

Sequences were screened against the UniVec database. In total, 176 high quality EST sequences were obtained. These genes were all functionally annotated by blasting against the NCBI database, and 51 differentially expressed genes were annotated. Of these, 24 were upregulated (Table 2 ) and 27 down-regulated (Table 3 ).

\section{Gene ontology}

Gene ontology terms for the 176 unique sequences were obtained. Of these, 76 were upregulated, and 100 down-regulated. Sequence descriptions and GO numbers are summarized in Tables 4 and 5. Under the biological process GO terms in the reverse library, $5.83 \%$ were cellular processes involved in reproduction. In the forward library, $5.88 \%$ were involved in developmental processes; no sequences involved in reproduction were observed. In the molecular function category, $20 \%$ of genes in the reverse library, and $15 \%$ in the forward library, were classified to 
have structural molecule activities. As to the cellular component category, $9.65 \%$ in the reverse, and $11.33 \%$ in the forward libraries were related directly to intracellular function.

Table 2. Putative up-regulated genes in mature ovary.

\begin{tabular}{|c|c|c|c|}
\hline Sample No. & Homologous to genes in database & Organism & E-value \\
\hline D08-A6 & Zinc proteinase Mpc1 ABD65301 & Litopenaeus vannamei & $8.83 e-103$ \\
\hline A07-A5 & Adenine nucleotide translocase AEZ68611 & Litopenaeus vannamei & $5.51 e-57$ \\
\hline A03-C9 & Alpha 2-macroglobulin AEC50080 & Pacifastacus leniusculus & $3.37 \mathrm{e}-27$ \\
\hline F09-C12 & Chromosome 4 open reading frame 34 NP_001016053 & Xenopus (Silurana) tropicalis & $2.49 \mathrm{e}-22$ \\
\hline B03-B9 & Chymotrypsin-like serine proteinase ACC68669 & Fenneropenaeus chinensis & $3.76 e-104$ \\
\hline F10-D1 & Cytochrome b BAD16771 & Acromyrmex echinatior & $7.23 e-28$ \\
\hline G03-D8 & Cytochrome c oxidase polypeptide vb AFV69126 & Euphausia superba & $3.01 e-25$ \\
\hline C04-C3 & Eukaryotic translation initiation factor 3 subunit $d$ & Scylla paramamosain & $5.09 e-76$ \\
\hline F05-C8 & Fatty acid-binding protein AGO02161 & Scylla paramamosain & $5.79 e-22$ \\
\hline F12-B8 & Histone h3 CAH61024 & Philocelis karlingi & $1.35 e-44$ \\
\hline F06-C4 & Peritrophin-48 XP_001999045 & Acromyrmex echinatior & $8.36 \mathrm{e}-08$ \\
\hline E01-A9 & Low Density Lipoprotein Receptor XP_003690198 & Apis florea & $1.25 \mathrm{e}-14$ \\
\hline G02-D6 & Lectin c ADG85667 & Marsupenaeus japonicus & $1.41 e-08$ \\
\hline E09-B7 & NADH dehydrogenase XP_001654149 & Aedes aegypti & $4.95 e-26$ \\
\hline C05-D7 & Nfx1-type zinc finger-containing protein 1 EFX77800 & Daphnia pulex & $1.86 \mathrm{e}-33$ \\
\hline D05-A3 & Ovary development-related protein AAO73308 & Eriocheir sinensis & $2.02 e-52$ \\
\hline D09-A7 & Pdgf vegf-related factor 1 ADF87936 & Eriocheir sinensis & $7.44 \mathrm{e}-29$ \\
\hline B12-C12 & Polehole-like protein ACV60547 & Penaeus monodon & $4.43 e-09$ \\
\hline F04-C7 & Ovigerous-hair stripping substance AAP57670 & Chiromantes haematocheir & $4.24 \mathrm{e}-55$ \\
\hline F10-C12 & Receptor for activated protein kinase c1 ABU49887 & Penaeus monodon & $7.27 e-76$ \\
\hline D09-C7 & Short-chain dehydrogenease reductase EJY57385 & Aedes aegypti & $2.95 e-99$ \\
\hline F01-B11 & Splicing arginine serine-rich 7 ACO12997 & Lepeophtheirus salmonis & $8.06 e-37$ \\
\hline H07-C6 & von Willebrand factor AEC22817 & Macrobrachium nipponense & $7.87 e-31$ \\
\hline A04-G9 & Vitellogenin ABX89617 & Callinectes sapidus & $9.25 e-164$ \\
\hline
\end{tabular}

\begin{tabular}{|c|c|c|c|}
\hline Sample No. & Homologous to genes in database & Organism & E-value \\
\hline G04-H5 & Ribosomal protein AAV71145 & Callinectes sapidus & $4.08 e-153$ \\
\hline B02-G3 & 40s ribosomal protein s 12 CCE46016 & Nephrops norvegicus & $4.33 e-82$ \\
\hline A02-G7 & Alcohol dehydrogenase class-3-like XP_002738071 & Saccoglossus kowalevskii & $3.21 e-58$ \\
\hline E02-E5 & Aldehyde dehydrogenase XP_002425846 & Pediculus humanus corporis & $7.23 e-86$ \\
\hline G08-F1 & Anionic trypsin-2 precursor XP__002126930 & Ciona intestinalis & $1.50 \mathrm{e}-12$ \\
\hline G08-F5 & Arginine kinase AEZ68729 & Pagrus major & $7.99 \mathrm{e}-142$ \\
\hline D12-E3 & Carboxypeptidase a2 EFX80631 & Daphnia pulex & $7.49 \mathrm{e}-71$ \\
\hline H05-F6 & Activated protein kinase $\mathrm{C}$ receptor $\mathrm{AAO} 33307$ & Eriocheir sinensis & $1.32 \mathrm{e}-41$ \\
\hline B12-G6 & Chitinase ABY85409 & Scylla serrata s & $7.17 e-25$ \\
\hline G04-E6 & Chromadorea alt protein XP_002587162 & Branchiostoma floridae & $4.60 \mathrm{e}-08$ \\
\hline E02-E4 & Chymotrypsin-like protein AEE 25770 & Scylla paramamosain & $4.65 e-135$ \\
\hline G06-E9 & E3 sumo-protein ligase nse2 AFP23392 & Scylla paramamosain & $5.06 e-58$ \\
\hline A12-E3 & Ferritin AEK81609 & Portunus trituberculatus & $9.38 e-115$ \\
\hline $\mathrm{C} 07-\mathrm{H} 4$ & Glutathione s-transferase mu 3 XP_003444865 & Oreochromis niloticus & $7.07 e-104$ \\
\hline A11-F4 & Heme binding protein 2 BAA99544 & Amphibalanus amphitrite & $1.22 \mathrm{e}-08$ \\
\hline F04-F12 & Hemocyanin subunit 6 AAA96966 & Metacarcinus magister & $3.34 \mathrm{e}-100$ \\
\hline F02-F9 & Intracellular fatty acid binding protein ABE77153 & Pacifastacus leniusculus & $1.60 \mathrm{e}-04$ \\
\hline $\mathrm{F} 12-\mathrm{H} 1$ & Liver fatty acid binding protein ADP05225 & Eriocheir sinensis & $3.13 e-52$ \\
\hline H09-F12 & Macrophage mannose receptor 1-like AAX63905 & Fenneropenaeus chinensis & $1.15 e-05$ \\
\hline H02-F1 & Myosin regulatory light chain 2 smooth muscle ACY 66440 & Scylla paramamosain & $4.02 \mathrm{e}-106$ \\
\hline $\mathrm{H} 10-\mathrm{C} 2$ & Comm domain-containing protein 2 EGI62544 & Scylla paramamosain & $1.97 e-30$ \\
\hline A03-E7 & Peroxiredoxin 6 ACJ53746 & Scylla paramamosain & $1.85 e-22$ \\
\hline E09-F10 & Prefoldin subunit 5 BAM 18170 & Papilio xuthus & $3.25 e-56$ \\
\hline F05-G1 & Proteasome beta 4 subunit EFX84494 & Daphnia pulex & $9.18 \mathrm{e}-35$ \\
\hline B05-G7 & Vitelline membrane outer layer protein 1 homolog NP_493786 & Caenorhabditis elegans & $1.42 \mathrm{e}-15$ \\
\hline H08-F11 & Zinc finger protein-like $2.09 \mathrm{E}-17$ & Saccoglossus kowalevskii & $2.09 e-17$ \\
\hline C03-G9 & Zinc finger matrin-type protein 5 XP 002596619 & Branchiostoma floridae & $3.73 \mathrm{e}-17$ \\
\hline
\end{tabular}




\begin{tabular}{|c|c|c|c|}
\hline Annotation & GO No. & EST No. & Percentage (\%) \\
\hline Cellular component & GO:0005575 & 49 & 17.60 \\
\hline Intracellular part & GO:0044424 & 39 & 14.42 \\
\hline Cell part & GO:0044464 & 40 & 14.01 \\
\hline Intracellular & GO:0005622 & 40 & 9.65 \\
\hline Cell & GO:0005623 & 40 & 8.40 \\
\hline Intracellular organelle part & GO:0044446 & 22 & 8.37 \\
\hline Macromolecular complex & GO:0032991 & 27 & 7.61 \\
\hline Extracellular region & GO:0005576 & 13 & 7.53 \\
\hline Organelle & GO:0043226 & 30 & 6.28 \\
\hline Extracellular region part & GO:0044421 & 9 & 5.88 \\
\hline Plasma membrane & GO:0005886 & 8 & 5.65 \\
\hline Organelle part & GO:0044422 & 22 & 5.40 \\
\hline Membrane & GO:0016020 & 13 & 5.34 \\
\hline Non-membrane-bounded organelle & GO:0043228 & 24 & 5.28 \\
\hline Extracellular space & GO:0005615 & 5 & 5.00 \\
\hline Molecular function & GO:0003674 & 71 & 26.58 \\
\hline Structural molecule activity & GO:0005198 & 20 & 20.00 \\
\hline Binding & GO:0005488 & 35 & 15.78 \\
\hline Structural constituent of ribosome & GO:0003735 & 20 & 20.00 \\
\hline Lipid transporter activity & GO:0005319 & 12 & 12.00 \\
\hline Protein binding & GO:0005515 & 13 & 9.08 \\
\hline Substrate-specific transporter activity & GO:0022892 & 12 & 7.20 \\
\hline Transporter activity & GO:0005215 & 13 & 5.32 \\
\hline Hydrolase activity & GO:0016787 & 19 & 5.13 \\
\hline RNA binding & GO:0003723 & 9 & 5.08 \\
\hline Ribosome binding & GO:0043022 & 5 & 5.00 \\
\hline Laminin binding & GO:0043236 & 5 & 5.00 \\
\hline Catalytic activity & GO:0003824 & 28 & 5.00 \\
\hline Biological process & GO:0008150 & 67 & 16.69 \\
\hline Gene expression & GO:0010467 & 26 & 19.31 \\
\hline Protein metabolic process & GO:0019538 & 37 & 17.28 \\
\hline Cellular process & GO:0009987 & 37 & 17.00 \\
\hline Cellular macromolecule metabolic process & GO:0044260 & 27 & 15.48 \\
\hline Cellular biosynthetic process & GO:0044249 & 25 & 14.40 \\
\hline Macromolecule metabolic process & GO:0043170 & 39 & 14.23 \\
\hline Metabolic process & GO:0008152 & 48 & 13.06 \\
\hline Primary metabolic process & GO:0044238 & 39 & 12.24 \\
\hline Macromolecule biosynthetic process & GO:0009059 & 25 & 11.16 \\
\hline Transport & GO:0006810 & 29 & 10.09 \\
\hline Biosynthetic process & GO:0009058 & 25 & 8.64 \\
\hline Cellular metabolic process & GO:0044237 & 30 & 8.39 \\
\hline Lipid localization & GO:0010876 & 12 & 7.20 \\
\hline Establishment of localization & GO:0051234 & 29 & 6.06 \\
\hline Cellular process involved in reproduction & GO:0048610 & 11 & 5.83 \\
\hline Oxidation-reduction process & GO:0055114 & 7 & 5.56 \\
\hline Macromolecule localization & GO:0033036 & 22 & 5.01 \\
\hline
\end{tabular}

\section{Metabolic pathway annotation}

Sixty-four metabolic pathways were annotated, including 20 in up-regulated and 44 in down-regulated genes (Table 6). Four pathways (oxidative phosphorylation, carbon metabolism, fatty acid degradation, and protein digestion and absorption) were suggested to be involved in ovarian development.

\section{Transcription of selected genes by real-time PCR}

Expression of eight differential genes, including four up-regulated and four down-regulated genes, were detected by quantitative real-time RT-PCR (Figure 1). Most of the detected genes 
were showed similar expression characterization to the result of SSH library, except genes PLP and LFAB, which showed insignificant differences in mature ovary and immature ovary.

\begin{tabular}{|c|c|c|c|}
\hline Annotation & GO No. & EST No. & Percentage (\%) \\
\hline Cellular component & GO:0005575 & 41 & 17.44 \\
\hline Cell & GO:0005623 & 37 & 14.73 \\
\hline Intracellular part & GO:0044424 & 34 & 13.88 \\
\hline Macromolecular complex & GO:0032991 & 25 & 12.12 \\
\hline Intracellular & GO:0005622 & 35 & 11.33 \\
\hline Cell part & GO:0044464 & 36 & 11.21 \\
\hline Protein complex & GO:0043234 & 10 & 10.00 \\
\hline Non-membrane-bounded organelle & GO:0043228 & 23 & 8.64 \\
\hline Organelle & GO:0043226 & 32 & 7.01 \\
\hline Molecular function & GO:0003674 & 53 & 36.95 \\
\hline Binding & GO:0005488 & 25 & 20.15 \\
\hline Catalytic activity & GO:0003824 & 25 & 15.30 \\
\hline Structural molecule activity & GO:0005198 & 15 & 15.00 \\
\hline Hydrolase activity & GO:0016787 & 18 & 12.48 \\
\hline Peptidase activity & GO:0008233 & 9 & 9.00 \\
\hline Protein binding & GO:0005515 & 10 & 8.72 \\
\hline Nucleic acid binding & GO:0003676 & 13 & 8.00 \\
\hline RNA binding & GO:0003723 & 10 & 8.00 \\
\hline Nucleotide binding & GO:0000166 & 7 & 7.00 \\
\hline Transporter activity & GO:0005215 & 7 & 6.13 \\
\hline Biological process & GO:0008150 & 50 & 60.17 \\
\hline Metabolic process & GO:0008152 & 36 & 24.81 \\
\hline Protein metabolic process & GO:0019538 & 27 & 16.12 \\
\hline Regulation of biological process & GO:0050789 & 15 & 16.08 \\
\hline Primary metabolic process & GO:0044238 & 28 & 14.69 \\
\hline Catabolic process & GO:0009056 & 14 & 14.00 \\
\hline Transport & GO:0006810 & 15 & 13.20 \\
\hline Cellular process & GO:0009987 & 32 & 11.36 \\
\hline Gene expression & GO:0010467 & 17 & 10.20 \\
\hline Macromolecule metabolic process & GO:0043170 & 27 & 10.03 \\
\hline Cellular metabolic process & GO:0044237 & 25 & 9.68 \\
\hline Biological regulation & GO:0065007 & 15 & 9.65 \\
\hline Nucleobase-containing compound metabolic process & GO:0006139 & 8 & 8.36 \\
\hline Establishment of localization & GO:0051234 & 15 & 7.92 \\
\hline Cellular component organization & GO:0016043 & 9 & 7.66 \\
\hline Cellular macromolecule metabolic process & GO:0044260 & 17 & 6.72 \\
\hline Biosynthetic process & GO:0009058 & 20 & 6.67 \\
\hline Macromolecule biosynthetic process & GO:0009059 & 17 & 6.12 \\
\hline Cellular biosynthetic process & GO:0044249 & 17 & 6.12 \\
\hline Developmental process & GO:0032502 & 6 & 5.88 \\
\hline
\end{tabular}

\section{DISCUSSION}

Understanding gonad development is necessary to control reproductive maturation in the economically important species (Leelatanawit et al., 2009). As an important measure for understanding molecular mechanisms of physiological traits such as gland maturation and sex differentiation, a large number of EST projects on crustaceans have been recently reported (Zou et al., 2011). Suppressive subtractive hybridization was reported to be an applicable technique to many molecular genetic and positional cloning studies, for the identification of disease, developmental, tissue specific, or other differentially expressed genes (Diatchenko et al., 1996). Screening of an ovarian cDNA library for shrimp, Marsupenaeus japonicus, using SSH-enriched probes has been reported (Zhang et al., 2007), and more than 20 differentially expressed genes were identified in 
ovary and testis. To date, no cDNA library has been constructed to select development-related genes in $P$. trituberculatus. In the present study, differentially expressed genes in mature and immature ovary from $P$. trituberculatus were analyzed using SSH. Expression characterization of 8 genes was also carried out by quantitative real-time PCR, which confirmed that SSH is valid for studies of mechanisms of ovarian development in the swimming crab.

\section{Gene ontology analysis and metabolic pathway annotation}

Functional similarities among genes are the strength of the relatedness of gene functions (Chen et al., 2012). Gene ontology was useful for analysis of identified ESTs. In the current study, $5.83 \%$ of the differential genes from cellular process is involved in reproduction in reverse subtraction cDNA library, and $5.88 \%$ were involved in developmental process in forward library. KEGG annotation indicated that transcription of genes related to nutrition metabolism pathways, such as oxidative phosphorylation, carbon metabolism, fatty acid degradation, protein digestion and absorption, were reduced in the mature ovary, which is consistent with the requirement by the immature ovary of more nutrition for development.

\section{Identification of differentially expressed ESTs}

Cholesterol is required for the synthesis of cell membranes and its uptake is mediated by the low-density lipoprotein (LDL) receptor (Goldstein et al., 1979). Fatty acid-binding protein (FABP) is involved in the uptake and intracellular translocation of long-chain fatty acids (Glatz and van der Vusse, 1996). Additionally, FABPs have been reported to govern transcriptional activities of their ligands by targeting them to cognate peroxisome proliferator-activated receptors (PPARs) in the nucleus in a receptor- and ligand-selective manner, thereby enabling PPARs to exert their biological functions (Tan et al., 2002). Peroxisome proliferator-activated receptors regulate transcription of genes involved in glucose uptake, lipid metabolism, and inflammation (Lincoff et al., 2013). The up-regulation of LDL receptor and FABP in the mature ovary may provide materials for the coming embryonic development.

Carboxypeptidases catalyze hydrolysis of C-terminal amino acids from their substrates and are involved in the digestion process (Gomis-Ruth, 2008). Members of the alcohol dehydrogenase $(\mathrm{ADH})$ family catalyze the reversible oxidation of a wide variety of primary and secondary alcohols into corresponding aldehydes and ketones (Dołęga, 2010). Aldehyde dehydrogenases (ALDH) are a group of enzymes that catalyze the oxidation of a broad spectrum of aliphatic and aromatic aldehydes (Lindahl, 1992). Arginine kinase plays a key role in the coupling of energy production and utilization in animals (Ellington, 2001). In the present study, down-regulation of carboxypeptidase, alcohol dehydrogenase class, carboxypeptidase, aldehyde dehydrogenase and arginine kinase transcription, in the mature ovary, is consistent with results of metabolism pathway annotation.

COMM domain-containing protein was demonstrated to associate with with NF-kB and inhibit its transcriptional activity (Burstein et al., 2005). NF-KB was demonstrated to play major role in the activation of numerous genes involved in the function and development of immune system (Baeuerle and Henkel, 1994, Baldwin, 1996). Chymotrypsin-like serine proteinase is involved in immune defense reactions against bacteria in Drosophila (de Morais Guedes et al., 2005), and innate immune reactions in Fenneropenaeus chinensis (Shi et al., 2008). Alpha 2-macroglobulin ( $\alpha 2-\mathrm{M}$ ) is protease inhibitor that can neutralize pathogenic proteases that contribute to pathogen virulence (Lin et al., 2008). 
Alpha 2-macroglobulinis an important innate immune element in crustaceans (Rattanachai et al., 2004). Lectins comprise a structurally diverse class of proteins characterized by their ability to bind carbohydrates with considerable specificity and play roles in biological-recognition events (Rini, 1995). The different transcript characterization of immune related genes indicated that the innate immune system was involved in ovarian maturation.

\section{Identification of ovarian development-related ESTs}

The development of oocytes is a series of complex cellular events, in which differential genes are temporally and spatially expressed to ensure proper development of oocytes, or to store transcripts and proteins as maternal factors for early embryogenesis (Qiu et al., 2005). Myosin II, expressed in non-muscle tissues, plays a central role in cell adhesion, migration and division; myosin regulatory light chains are essential for maintaining the integrity of myosin II, and are critical for cell structure and dynamics (Park et al., 2011). Polehole-like protein in Penaeus monodon was appears to be involved in ovarian development (Klinbunga et al., 2009). The upregulated expression level of polehole-like protein in mature ovary of $P$. trituberculatus indicated its involvement in the ovarian development of the swimming crab. Histone h3 of Drosophila was reported to be necessary for fertility (Sakai et al., 2009), suggesting an important role in gametogenesis (Filipescu et al., 2013). Up-regulated expression level of histone h3 in the mature ovary may be beneficial to the developmental of the ovary. Ovigerous-hair stripping substance (OHSS), released by the embryo upon hatching (Gusev et al., 2004), plays a role in the stripping of the embryo attachment system from the maternal ovigerous hairs following hatching, in preparation for the next clutch of embryos (Saigusa, 1995). Ovary development related protein was firstly cloned in Eriocheir sinensis (AEC22817), and was up-regulated in the mature ovary. Peritrophic matrix (or peritrophin) was involved in the digestive process through its role in partitioning of digestive enzymes and semi-digested food between the endo- and ecto-peritrophic spaces (Vuocolo et al., 2001). Peritrophic matrix plays key roles in the intestinal biology of insects, and might protect the midgut epithelium from mechanical damage and insults from pathogens and toxins (Lehane, 1997). In addition, peritrophin was demonstrated to be involved in ovary development of the red swamp crayfish Procambarus clarkii (Shui et al., 2012). In the present study, 6 genes involved in ovarian development were found to be up-regulated in mature ovary.

Vitellogenin $(\mathrm{Vg})$ is the precursor of vitellin, the major yolk protein stored in crustacean oocytes that provides free amino acids, lipids, carbohydrates, carotenoids, and minerals to the developing embryo and larva (Byrne et al., 1989). It is mainly expressed in the hepatopancreas, and trace amounts of transcript have been found in the ovary during vitellogenesis in $P$. trituberculatus (Byrne et al., 1989). Increased vitellogenin mRNA in the mature ovary of $P$. trituberculatus might be useful for ovary development.

Vitelline membrane outer layer protein is the major component of the outer layer of vitelline membrane (Kido et al., 1992). Decreased transcription of this protein in the mature ovary may result from consumption of mRNAs for protein synthesis, consistent with the phenomenon that more eggs are found in mature than immature ovaries.

In conclusion, SSH libraries were constructed from mature and immature ovaries of $P$. trituberculatus. Four annotated pathways were suggested to be involved in ovarian development. Gene ontology analysis indicated that in the reverse subtraction cDNAlibrary, $5.83 \%$ of the differential expressed genes in cellular processes involved in reproduction, and $5.88 \%$ in the forward library 
were involved in developmental processes. Six genes (myosin II expressed, polehole-like protein, histone h3, ovigerous-hair stripping substance (OHSS), peritrophin 48 and ovary development related protein) were up-regulated in mature ovary. This study provides new insights for further studies onovarian development in the swimming crab.

\section{Conflicts of interest}

The authors declared that they have no conflicts of interest to this work.

\section{ACKNOWLEDGMENTS}

The authors sincerely thank the many people who assisted with the work and manuscript. Research supported by the National Natural Science Foundation of China (grant \#41106123, \#41376150, and \#41476124); National High Technology Research and Development Program of China (grant \#2012AA10A409); Agriculture Science Technology Achievement Transformation Fund (\#2014GB2C220151); Ningbo Key Programs for Science and Technology Development (\#2013C11017); China Spark Program (\#2013GA701002; and K.C. Wong Magna Fund, Ningbo University.

\section{REFERENCES}

Baeuerle PA and Henkel T (1994). Function and activation of NF-kappa B in the immune system. Annu. Rev. Immunol. 12: 141-179.

Baldwin AS Jr (1996). The NF-kappa B and I kappa B proteins: new discoveries and insights. Annu. Rev. Immunol. 14: 649683.

Byrne BM, Gruber M and Ab G (1989). The evolution of egg yolk proteins. Prog. Biophys. Mol. Biol. 53: 33-69.

Chen X, Yang R, Xu J, Ma H, et al. (2012). A sensitive method for computing GO-based functional similarities among genes with 'shallow annotation'. Gene 509: 131-135.

de Morais Guedes S, Vitorino R, Domingues R, Tomer K, et al. (2005). Proteomics of immune-challenged Drosophila melanogaster larvae hemolymph. Biochem. Biophys. Res. Commun. 328: 106-115.

Diatchenko L, Lau YF, Campbell AP, Chenchik A, et al. (1996). Suppression subtractive hybridization: a method for generating differentially regulated or tissue-specific cDNA probes and libraries. Proc. Natl. Acad. Sci. U. S. A. 93: 6025-6030.

Dołęga A (2010). Alcohol dehydrogenase and its simple inorganic models. Coord. Chem. Rev. 254: 916-937.

Duguid JR and Dinauer MC (1990). Library subtraction of in vitro cDNA libraries to identify differentially expressed genes in scrapie infection. Nucleic Acids Res. 18: 2789-2792.

Ellington WR (2001). Evolution and physiological roles of phosphagen systems. Annu. Rev. Physiol. 63: 289-325.

Elner RW and Beninger PG (1992). The reproductive biology of snow crab, Chionoecetes opilio: a synthesis of recent contributions. Am. Zool. 32: 524-533.

Filipescu D, Szenker E and Almouzni G (2013). Developmental roles of histone H3 variants and their chaperones. Trends Genet. 29:630-640.

Goldstein JL, Anderson RG and Brown MS (1979). Coated pits, coated vesicles, and receptor-mediated endocytosis. Nature 279: 679-685.

Gomis-Ruth FX (2008). Structure and mechanism of metallocarboxypeptidases. Crit. Rev. Biochem. Mol. Biol. 43: $319-345$.

Glatz JF and van der Vusse GJ (1996). Cellular fatty acid-binding proteins: their function and physiological significance. Prog. Lipid Res. 35: 243-282.

Gusev O, Ikeda H, Okochi T, Lee JM, et al. (2004). Purification and cDNA cloning of the ovigerous-hair stripping substance (OHSS) contained in the hatch water of an estuarine crab Sesarma haematocheir. J. Exp. Biol. 207: 621-632.

Kido S, Morimoto A, Kim F and Doi Y (1992). Isolation of a novel protein from the outer layer of the vitelline membrane. Biochem. J. 286: 17-22.

Klinbunga S, Sittikankaew K, Yuvanatemiya V, Preechaphol R, et al. (2009). Molecular cloning and expression analysis of ovary-specific transcript 1 (Pm-OSTI) of the giant tiger shrimp, Penaeus monodon. Zool. Sci. 26: 783-790. 
Lehane MJ (1997). Peritrophic matrix structure and function. Annu. Rev. Entomol. 42: 525-550.

Leelatanawit R, Sittikankeaw K, Yocawibun P, Klinbunga S, et al. (2009). Identification, characterization and expression of sex-related genes in testes of the giant tiger shrimp Penaeus monodon. Comp. Biochem. Physiol. A Mol. Integr. Physiol. 152: 66-76.

Lin YC, Vaseeharan B and Chen JC (2008). Molecular cloning and phylogenetic analysison alpha2-macroglobulin (alpha2-M) of white shrimp Litopenaeus vannamei. Dev. Comp. Immunol. 32: 317-329.

Lincoff AM, Tardif JC, Neal B, Nicholls SJ, et al. (2013). Evaluation of the dual peroxisome proliferator-activated receptor $a / \mathrm{Y}$ agonist aleglitazar to reduce cardiovascular events in patients with acute coronary syndrome and type 2 diabetes mellitus: Rationale and design of the AleCardio trial. Am. Heart J. 166: 429-434.

Lindahl R (1992). Aldehyde dehydrogenases and their role in carcinogenesis. Crit. Rev. Biochem. Mol. Biol. 27: 283-335.

Liu Y, Cui Z, Luan W, Song C, et al. (2011). Three isoforms of anti-lipopolysaccharide factor identified from eyestalk cDNA library of swimming crab Portunus trituberculatus. Fish Shellfish Immunol. 30: 583-591.

Park I, Han C, Jin S, Lee B, et al. (2011). Myosin regulatory light chains are required to maintain the stability of myosin II and cellular integrity. Biochem. J. 434: 171-180.

Qiu GF, Yamano K and Unuma T (2005). Cathepsin C transcripts are differentially expressed in the final stages of oocyte maturation in kuruma prawn Marsupenaeus japonicus. Comp. Biochem. Physiol. B Biochem. Mol. Biol. 140: 171-181.

Rattanachai A, Hirono I, Ohira T, Takahashi Y, et al. (2004). Molecular cloning and expression analysis of alpha 2-macroglobulin in the kuruma shrimp, Marsupenaeus japonicus. Fish Shellfish Immunol. 16: 599-611.

Rini JM (1995). Lectin structure. Annu. Rev. Biophys. Biomol. Struct. 24: 551-577.

Sakai A, Schwartz BE, Goldstein S and Ahmad K (2009). Transcriptional and developmental functions of the H3.3 histone variant in Drosophila. Curr. Biol. 19: 1816-1820.

Saigusa M (1995). Bioassay and preliminary characterization of ovigerous-hair stripping substance (OHSS) in hatch water of crab larvae. Biol. Bull. 189: 175-184.

Shi XZ, Zhao XF and Wang JX (2008). Molecular cloning and expression analysis of chymotrypsin-like serine protease from the Chinese shrimp, Fenneropenaeus chinensis. Fish Shellfish Immunol. 25: 589-597.

Shui Y, Guan ZB, Xu ZH, Zhao CY, et al. (2012). Proteomic identification of proteins relevant to ovarian development in the red swamp crayfish Procambarus clarkii. Aquaculture 370-371: 14-18.

Tan NS, Shaw NS, Vinckenbosch N, Liu P, et al. (2002). Selective cooperation between fatty acid binding proteins and peroxisomeproliferator-activated receptors in regulating transcription. Mol. Cell. Biol. 22: 5114-5127.

Vuocolo T, Eisemann CH, Pearson RD, Willadsen P, et al. (2001). Identification and molecular characterisation of a peritrophin gene, peritrophin-48, from the myiasis fly Chrysomya bezziana. Insect Biochem. Mol. Biol. 31: 919-932.

Wu XG, Yao GG, Yang XZ, Cheng YX, et al. (2007). A study on the ovarian development of Portunus trituberculatus in East China Sea during the first reproductive cycle. Acta. Oceanol. Sin. 29: 120-127.

Xie Y, Li F, Wang B, Li S, et al. (2010). Screening of genes related to ovarian development in Chinese shrimp Fenneropenaeus chinensis by suppression subtractive hybridization. Comp. Biochem. Physiol. Part D Genomics Proteomics 5: 98-104.

Zhang Z, Wang Y, Jiang Y, Lin P, et al. (2007). Ribosomal protein L24 is differentially expressed in ovary and testis of the marine shrimp Marsupenaeus japonicus. Comp. Biochem. Physiol. B Biochem. Mol. Biol. 147: 466-474.

Zou Z, Zhang Z, Wang Y, Han K, et al. (2011). EST analysis on the gonad development related organs and microarray screen for differentially expressed genes in mature ovary and testis of Scylla paramamosain. Comp. Biochem. Physiol. Part $D$ Genomics Proteomics 6: 150-157. 